\title{
Enhancement of electric and magnetic wave fields at density gradients
}

\author{
A. Reiniusson ${ }^{1}$, G. Stenberg ${ }^{1,2}$, P. Norqvist ${ }^{1}$, A. I. Eriksson ${ }^{2}$, and K. Rönnmark ${ }^{1}$ \\ ${ }^{1}$ Department of Physics, Umeå University, Umeå, Sweden \\ ${ }^{2}$ Swedish Institute of Space Physics, Uppsala, Sweden
}

Received: 26 August 2005 - Accepted: 20 December 2005 - Published: 7 March 2006

\begin{abstract}
We use Freja satellite data to investigate irregular small-scale density variations. The observations are made in the auroral region at about $1000-1700 \mathrm{~km}$. The density variations are a few percent, and the structures are found to be spatial down to a scale length of a few ion gyroradii. Irregular density variations are often found in an environment of whistler mode/lower hybrid waves and we show that at the density gradients both the electric and magnetic wave fields are enhanced.
\end{abstract}

Keywords. Magnetospheric physics (Auroral phenomena) - Space plasma physics (Wave-particle interactions; Waves and instabilities)

\section{Introduction}

Whistler mode and lower hybrid waves are frequently observed on auroral field lines. Sudden enhancements of the wave power in these modes are often associated with smallscale density variations. One common type of small-scale density structure was initially observed at altitudes below 1500 km (e.g. Fejer and Kelley, 1980; Sagalyn et al., 1974). These structures have scale lengths down to about the ion gyro radius and are referred to as spatial irregularities: the density profile varies irregularly and the density depletions are generally a few percent. Holmgren and Kintner (1990) use data from the Viking satellite to show the existence of such irregular static structures also at higher altitudes and latitudes. Other observations of irregular structures have been made by Temerin (1978), using S3-3 data, and by Delory et al. (1997), using Alaska ' 93 sounding rocket data. Delory et al. (1997) show that waves with very short wavelengths can be found on small-scale density gradients, while waves between the structures have much longer wavelengths. Hence, they suggest that mode conversion occurs on the density gradients.

Correspondence to: A. Reiniusson

(annie.reiniusson@space.umu.se)
Another well-known type of density depletion is the socalled Lower Hybrid Cavity (LHC) or Lower Hybrid Solitary Structure (LHSS). LHCs are elongated structures aligned with the background magnetic field observed both by rockets on low altitudes and by numerous satellites on altitudes up to $35000 \mathrm{~km}$ (Tjulin et al., 2003; Schuck et al., 2003 and references therein). They are found to be spatial structures (e.g. Knudsen et al., 1998) with a Gaussian-shaped density profile perpendicular to the ambient magnetic field. The width of a typical cavity is a few ion gyroradii and the density depletion is a few percent up to several tens of percent. Previous studies using data from the Freja satellite show clear enhancements of the electric wave power on the density gradients of LHCs, while no increases in the magnetic wave power are reported (e.g. Høymork et al., 2000; Dovner et al., 1994; Eriksson et al., 1994). These observations are consistent with theoretical models that predict electrostatic or almost electrostatic waves to be trapped in the cavities (Schuck et al., 2003; Hall et al., 2004). However, recent rocket observations indicate that LHCs sometimes can be associated with electromagnetic signatures (D. Knudsen, private communication).

The LHC phenomenon is also investigated in laboratories (e.g. Rosenberg and Gekelman, 2001). However, in contrast to most space observations, the waves detected in the cavities examined in these experiments are electromagnetic. This obvious difference might, at least partly, be due to the difficulties of setting up an experiment in the laboratory that resembles the space environment. There is an ongoing discussion about the proper parameter scaling between space and laboratory plasmas (Schuck et al., 2004; Schuck et al., 2003; Gekelman, 2004).

In this article we focus mainly on waves observed in association with irregular density variations. We study both magnetic and electric wave fields, and are especially interested in enhancements of the wave power at the density gradients. We characterize the plasma environment in which these types of density variations are found and determine the wavelengths and propagation direction of the surrounding whistler mode/lower hybrid waves. We also discuss the 
November 14, 1994

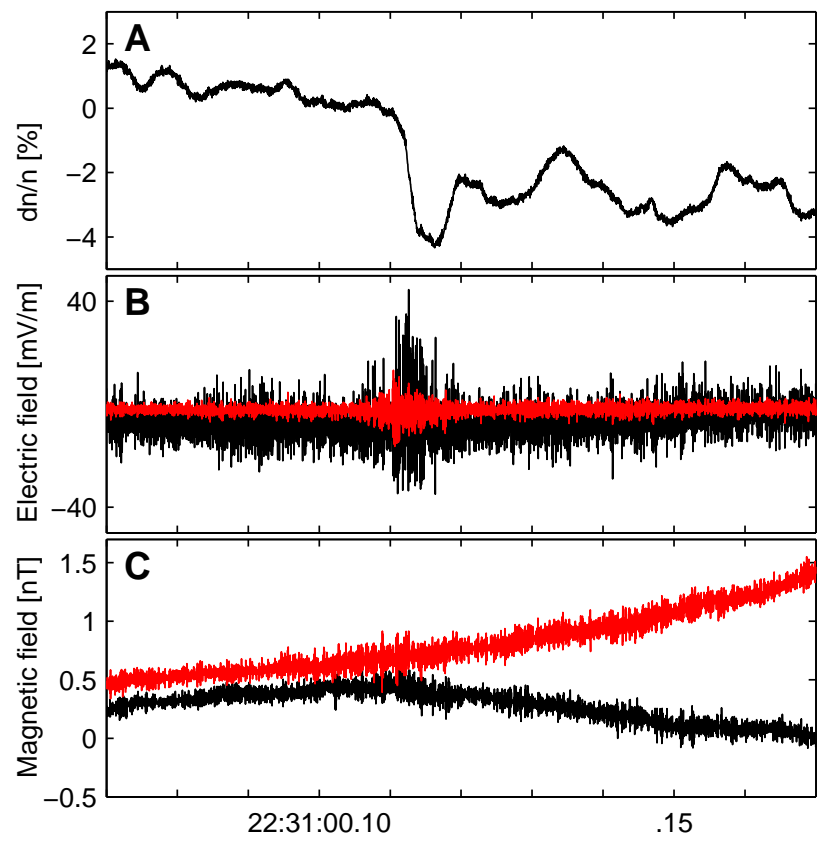

Fig. 1. Panel (A) shows the density variations observed along the satellite trajectory (perpendicular to $\boldsymbol{B}_{0}$ ). The two recorded electric wave field components are shown in panel (B). The black and red lines are the most perpendicular and the most parallel $\mathbf{E}$-field components, respectively. For both components we note a clear enhancement associated with the density gradient. In panel (C) corresponding magnetic wave fields are presented. Here the spin axis component (black line) has an angle of $77^{\circ}$ to $\boldsymbol{B}_{0}$ and the spin plane component (red line) has an angle of $43^{\circ}$ to $\boldsymbol{B}_{0}$. The increase in the magnetic wave activity is barely visible.

similarities and differences between irregular density variations and LHCs. As far as we are aware, this is the first satellite-based study detecting both magnetic and electric wave enhancements at small-scale density gradients.

The outline of this paper is as follows: first, we present two events where we find irregular density variations. They are examined in some detail in Sect. 3. A small statistical study based on 100 Freja orbits follows in Sect. 4. In Sect. 5 we investigate the wave activity surrounding both the irregular density variations and the LHCs.

\section{Instrumentation and method}

The Freja satellite was launched in October 1992 into an orbit with apogee $1756 \mathrm{~km}$, perigee $601 \mathrm{~km}$ and inclination $63^{\circ}$, and provided almost three years of data (Lundin et al., 1994b; Lundin et al., 1998). This paper is based on data from the Freja wave instrument (F4) (Holback et al., 1994), which included six electrostatic probes mounted on wire booms in the spin plane, as well as a three-axis search coil magnetometer. To achieve waveform resolution at high frequencies, a snapshot technique was used, where normally four signals from these sensors were sampled at 4096 samples/s in brief intervals (typically $0.75 \mathrm{~s}$ every $4 \mathrm{~s}$ ). At the same time, even shorter snapshots were sampled at 32768 samples/s. In what was known as "burst mode", up to five signals could be simultaneously sampled at 32768 samples/s for some $15 \mathrm{~s}$. These burst mode data, taken typically once or twice per day, form the basis of this study, as they allow for a detailed study of wave and plasma density variations.

The electrostatic probes could be used either in electric field mode, measuring voltage differences between probes fed with a constant bias frequency, or in Langmuir mode, sampling the current variations to a probe at constant bias voltage. For the plasma densities and density variation time scales we investigate here, the probe current variations are not significantly influenced by electric field fluctuations in a coupling capacity to the probe (Eriksson and Boström, 1998), and can be assumed to reflect variations in plasma density.

The ion data presented are obtained by the $\mathrm{F} 3 \mathrm{H}$ hot plasma instrument (Eliasson et al., 1994) and the electron data are from the electron spectrometer (F7) (Boehm et al., 1994). The flux gate magnetometer F2 provides the background magnetic field (Zanetti et al., 1994).

\section{Event studies}

Density variations of a few percent are common at the Freja altitude. The cigar-shaped LHCs are well known. In this paper we focus on another type of density structure, which we will refer to as irregular density variations. In contrast to LHCs these density depletions are generally asymmetric and more or less one-sided. Furthermore, LHCs are associated with clear enhancements in the electric field only, while in most cases both the electric and the magnetic wave activity increase at the gradients of irregular density variations.

\subsection{Event 1: 14 November 1994}

Figure 1 presents time series data recorded by Freja on 14 November 1994 . We show $0.2 \mathrm{~s}$ of data, including an example of the type of density variations we study. The event is observed at $1200-\mathrm{km}$ altitude in the auroral region (MLT: 17.9 h, CGLAT: $67^{\circ}$ ). In panel (A) the density variations along the satellite trajectory are displayed. For this case the spacecraft velocity is perpendicular to the background magnetic field, $\boldsymbol{B}_{0}$. At 22:31:00.11 UT the density rapidly drops about $4 \%$ but rises again about $0.01 \mathrm{~s}$ later. Hence, assuming a spatial structure and a satellite velocity of $7 \mathrm{~km} / \mathrm{s}$ the width of this density depletion is approximately $70 \mathrm{~m}$.

Panel (B) shows the two available electric field (E) components. The black line is the $\boldsymbol{E}$-field component most perpendicular to $\boldsymbol{B}_{0}$ while the red line depicts the component most parallel to $\boldsymbol{B}_{0}$. The angles between $\boldsymbol{B}_{0}$ and the electric field components are $23^{\circ}$ and $74^{\circ}$, respectively. An enhancement in the wave activity at the sharp gradient is clearly seen in both components. It can also be noted that the most 


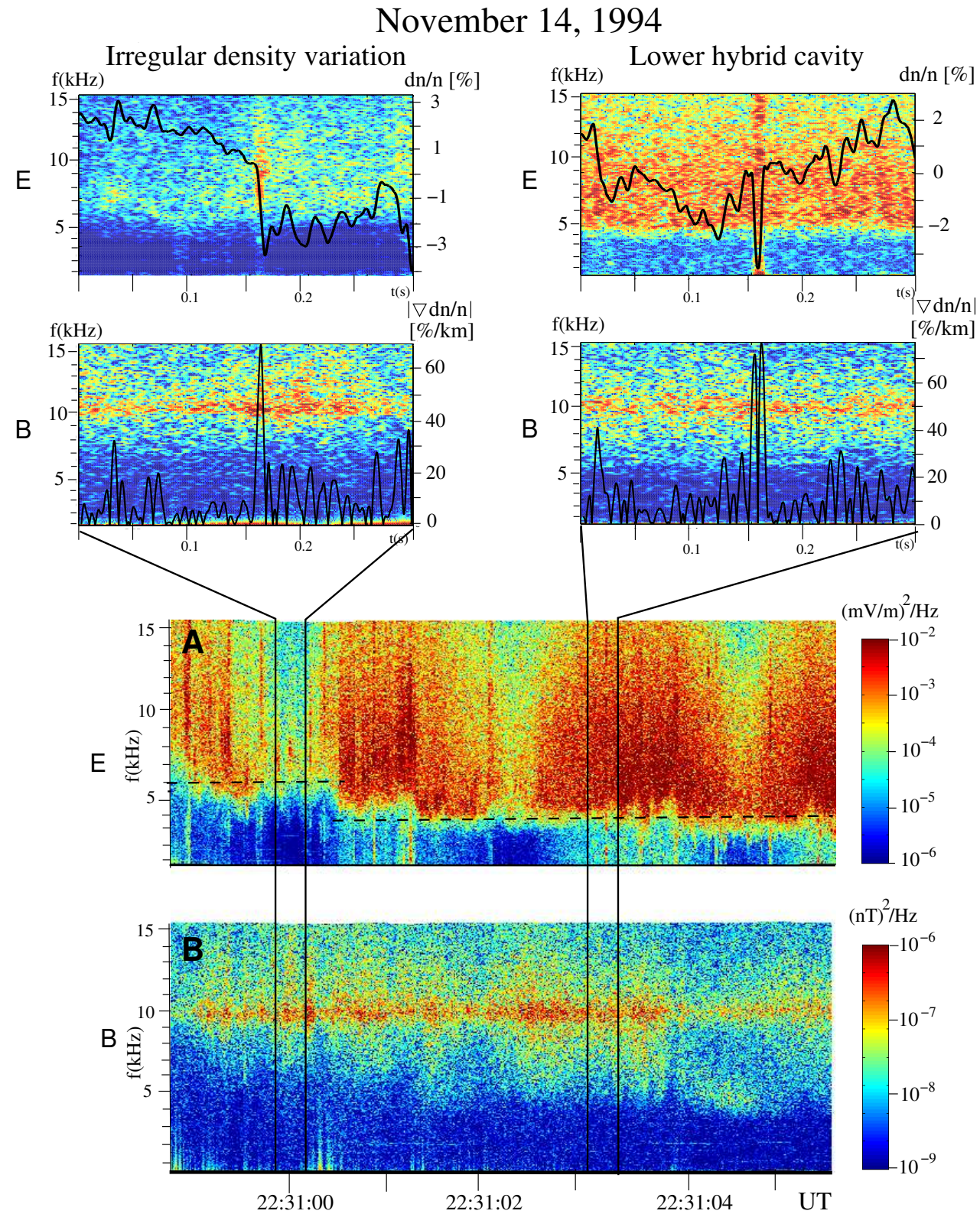

Fig. 2. The two large panels show 7-s long electric and magnetic field spectrograms from 14 November 1994. At 22:31:00.5 UT the total density changes from $7900 \mathrm{~cm}^{-3}$ to $3100 \mathrm{~cm}^{-3}$. The associated change in the lower hybrid cutoff frequency is marked with dashed lines in panel (A). Before the density drop irregular density variations are found and after are LHCs detected. Enlarged are $0.3 \mathrm{~s}$ from two parts of the burst. The two top-left panels show an irregular density variation event and the two top-right panels show an LHC event.

perpendicular component is larger throughout the time period shown.

In the bottom panel $(\mathrm{C})$, the two recorded magnetic field (B) components are displayed and we can see a tendency toward an increase in the wave field at the gradient. The black line is the spin axis component, with an angle to $\boldsymbol{B}_{0}$ of $77^{\circ}$, while the red line is a spin plane component with an angle of $43^{\circ}$ to $\boldsymbol{B}_{0}$. There is no obvious difference in amplitude between the two components.

Figure 2 shows a longer sequence from this burst. The two lower panels (A and B) display 7-s long electric and magnetic field spectrograms. At 22:31:00.5 UT there is a sudden decrease in density. The consequent decrease of the lower hybrid cutoff frequency is evident in the electric field spectrogram (dashed lines in panel A). This feature turns out to separate two regions; irregular density variations are found before and LHCs are detected after the density decrease. The two types of density variations are exemplified in Fig. 2 by the enlarged sections. In the two top-left panels the density and the absolute value of the density gradient (black lines) are shown on top of the electric and magnetic field spectrograms. Note that this is the same density variation as that 


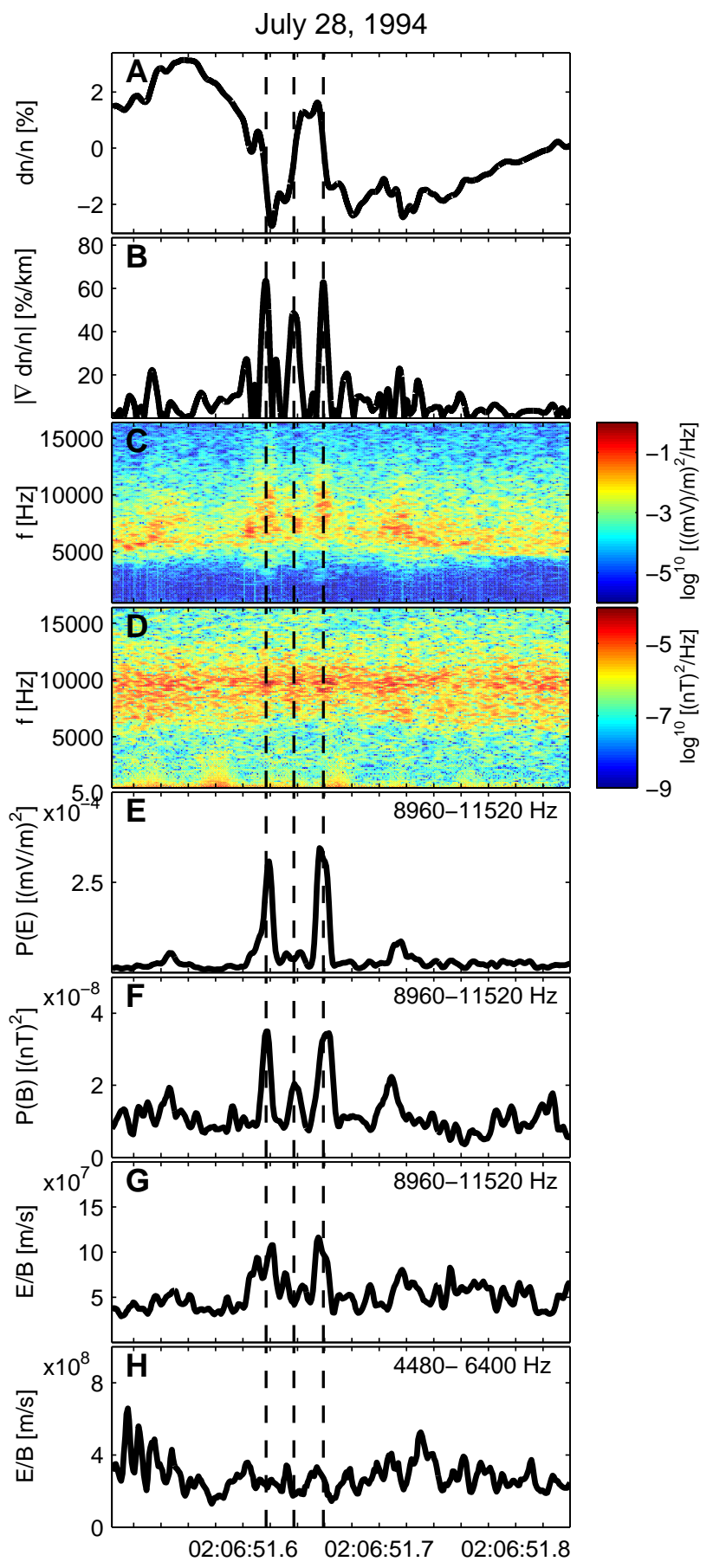

Fig. 3. Panel (A) shows the density profile. Panel (B) displays the absolute value of the gradient of the density. We assume spatial structures and the gradient is given in $\% / \mathrm{km}$. The electric and magnetic power spectral densities are plotted versus time and frequency in panels (C) and (D). The electric and magnetic wave powers in the frequency range 8960-11520 Hz are given in panels (E) and (F). Panels $(\mathbf{G})$ and $(\mathbf{H})$ present $E / B$ for the two frequency intervals, $8960-11520 \mathrm{~Hz}$ and $4480-6400 \mathrm{~Hz}$. shown in Fig. 1, but here the time series of the density is smoothed. The two top-right panels show a LHC from a few seconds later.

Throughout the $7 \mathrm{~s}$ shown in panel (B), there is a clear emission at $10 \mathrm{kHz}$. These waves probably have a different wave mode than the background electrostatic waves. They are more electromagnetic and propagate presumably at a smaller angle to $\boldsymbol{B}_{0}$. Since this emission seems rather unaffected by the local plasma properties, it may be suspected that the signal originates from a ground transmitter, such as the Omega stations transmitting at $10.2 \mathrm{kHz}$ (Green et al., 2005; Kimura et al., 2001). However, a closer examination reveals that the peak frequency varies between 9.5 and $11.5 \mathrm{kHz}$, and no amplitude variations corresponding to the $\sim 1$-s pulses in the Omega signals can be discerned. We cannot rule out that this signal originally came from a ground transmitter, but, as will be discussed later in Sect. 6, most of the results presented in this paper would not be affected if this were the case.

\subsection{Event 2: 28 July 1994}

Event 1 is a unique example in our study including both irregular density variations and LHCs. Our next event contains only irregular density variations. We have already seen in Fig. 1 that magnetic field enhancements associated with these variations are difficult to spot in time series data. Usually they are impossible to see, as the increase in amplitude is moderate and occurs in a limited frequency interval. However, an increase in the magnetic wave activity may still be evident in time frequency spectrograms. Figure 3 provides an example of irregular density variations, where no enhancements in the $\boldsymbol{B}$-field are seen in the time series data (not shown). We present $0.32 \mathrm{~s}$ of data recorded on 28 July 1994 (MLT: 18.8 h, CGLAT: $67^{\circ}$ ). Panel (A) shows the density variations for this time period. The dashed lines mark three sharp gradients, where the density changes a few percent in 5-10 ms. In panel (B) the absolute value of the gradient of the density profile is plotted. We again assume that the variations are spatial (cf. Sect. 3.2.2) and present the gradient in units of $\% / \mathrm{km}$. The three largest gradients have sizes of about 50 to $60 \% / \mathrm{km}$.

Panels (C) and (D) present the electric field and the magnetic field spectrograms, respectively. We use only one $\boldsymbol{E}$-field and one $\boldsymbol{B}$-field component. The electric field is here almost perpendicular to $\mathbf{B}_{\mathbf{0}}$ and the angle between $\boldsymbol{B}_{0}$ and the measured magnetic wave field component is $64^{\circ}$. Wave emissions occur in the frequency range $5 \mathrm{kHz}$ to at least $15 \mathrm{kHz}$. The sharp cutoff just below $5 \mathrm{kHz}$ is interpreted as the lower hybrid cutoff. In the $\boldsymbol{E}$-field spectrogram we see distinct enhancements in the spectral density at the marked gradients. The increases occur in a broad frequency band, ranging from just above the lower hybrid frequency cutoff to above $10 \mathrm{kHz}$. Enhancements in the $\boldsymbol{B}$-field are also clearly visible (panel D). However, these enhancements are only visible in the electromagnetic wave with frequencies around $10 \mathrm{kHz}$. 
July 28, 1994: Irregular density event

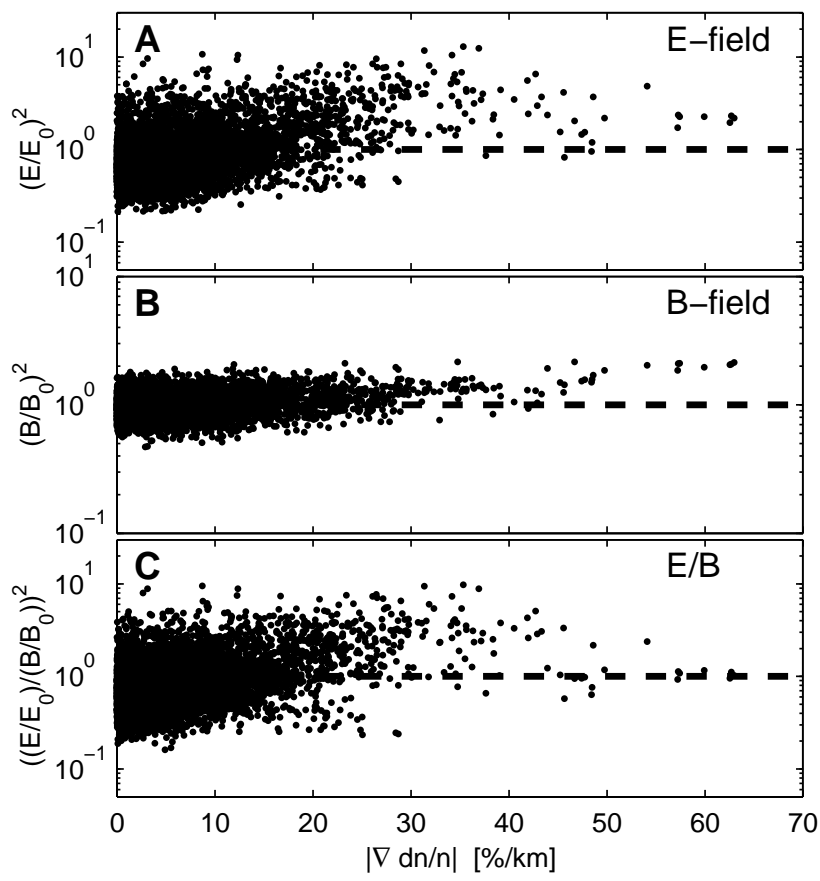

Fig. 4. Variations of the wave power for an irregular density variation case. The analysis is based on $10 \mathrm{~s}$ of data from 28 July 1994, containing the three gradients presented in Fig. 3. Panels (A) and (B) show variations in the electric and magnetic wave power versus the gradient of the density, $|\nabla d n / n|$. The wave power is normalized to the average wave power over the surrounding $0.5 \mathrm{~s}$. Hence, a ratio larger than one means that there is an enhancement in the wave power. For steep gradients $(|\nabla d n / n| \gtrsim 30 \% / \mathrm{km})$ we see clear enhancements of both the electric and the magnetic wave power. Panel (C) presents the variation in $E / B$ versus $|\nabla d n / n|$.

To estimate the increase in wave power at these gradients we integrate the power spectral densities in the frequency range $8960-11520 \mathrm{~Hz}$. Our frequency resolution is $128 \mathrm{~Hz}$ and the interval is chosen to cover the 10-kHz-enhancement. The results are displayed in panels (E) and (F). We see that the power in the $\boldsymbol{E}$-field is 10-20 times larger on the gradients (panel E), although the increase in power close to the lower hybrid cutoff, particularly at the middle gradient, is not captured by the summation. The power in $\boldsymbol{B}$ is roughly doubled (panel F).

The two bottom panels show $E / B$ for the two frequency intervals $8960-11520 \mathrm{~Hz}$ and $4480-6400 \mathrm{~Hz}$. The second interval is chosen to examine the wave activity around the lower hybrid frequency cutoff. In the higher frequency interval $E / B$ is larger at the gradients. We conclude that the electrostatic wave is more enhanced here than the electromagnetic wave. In the reference frequency interval, 4480$6400 \mathrm{~Hz}, E / B$ is not correlated with the gradients.

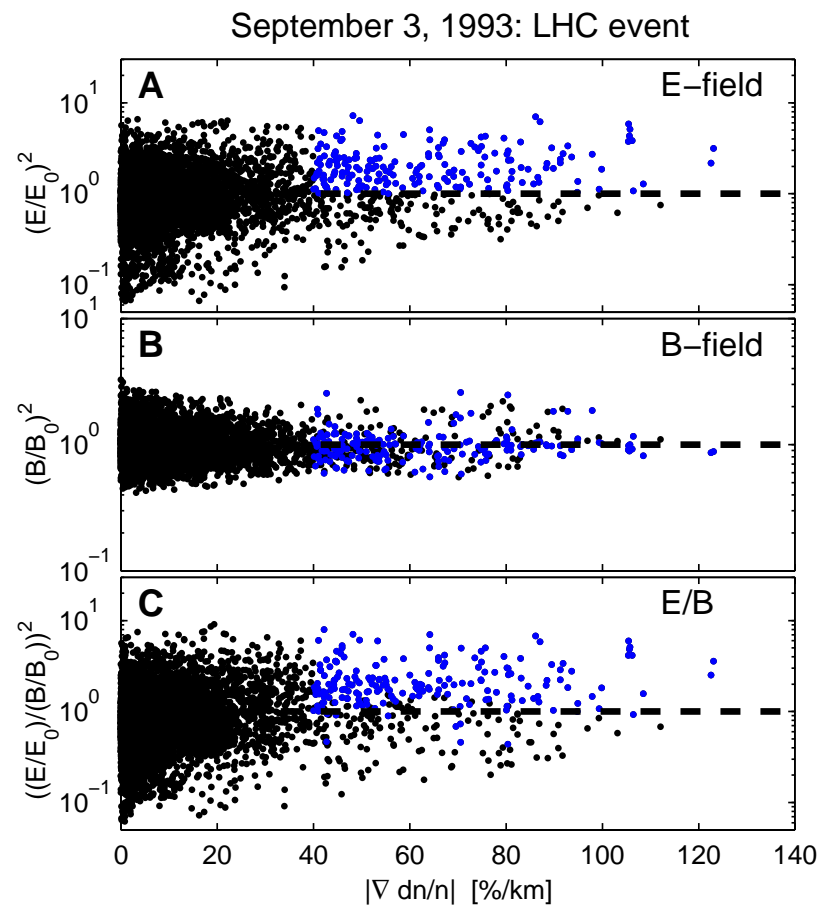

Fig. 5. Variations of the wave power for an LHC case. The analysis is based on $10 \mathrm{~s}$ of data observed on 3 September 1993. Analysis and panels are the same as in Fig. 4. All points with enhanced electric wave power and $|\nabla d n / n| \gtrsim 40 \% / \mathrm{km}$ are colored blue. From panel (B) we then see that there is no correlation between enhancements in the electric power and enhancements in the magnetic power.

\subsubsection{Irregular density variations versus LHCs}

To prove that the enhancements of magnetic fields at density gradients in Figs. 1-3 are not isolated events, we systematically analyze $10 \mathrm{~s}$ of data from this burst (28 July 1994). The entire time period is filled with irregular density variations. Following the procedure leading to panel (E) in Fig. 3 we compute an electric wave power $\left(\sim E^{2}\right)$ in the frequency range $8960-11520 \mathrm{~Hz}$. Then for each point in time the wave power is divided by a smoothed power $\left(\sim E_{0}^{2}\right)$, obtained using a floating average over 500 points $(\sim 0.5 \mathrm{~s})$. In panel (A) of Fig. $4\left(E / E_{0}\right)^{2}$ is plotted versus the magnitude of the density gradient. We see that for weak gradients we have little systematical variation of $\left(E / E_{0}\right)^{2}$, but for gradients $\gtrsim 30 \% / \mathrm{km}$ the electric wave fields are enhanced. A horizontal dashed line is drawn at $\left(E / E_{0}\right)^{2}=1$ for reference. The result of the corresponding analysis for the magnetic wave power is presented in panel (B). We see that the magnetic wave power is also clearly enhanced for gradients $\gtrsim 30 \% / \mathrm{km}$. The enhancement in the magnetic wave power is, however, only about two times, while the electric wave power is often enhanced up to ten times. Hence, the ratio $E / B$, displayed in panel (C), also becomes larger for steep gradients. 
We conclude that there is an enhanced wave activity at the gradients of irregular density variations. An increase in wave power is clear both for the electric and the magnetic wave fields. Simultaneously, the wave polarization changes, as we find a clear increase in $E / B$ at the gradients. However, no changes can be detected in the polarization parameters $E_{\perp} / E_{\|}$or $B_{\perp} / B_{\|}$(not shown).

Figure 5 shows the corresponding analysis for an LHC case. The $10 \mathrm{~s}$ of data used are observed on 3 September 1993, beginning at 04:45:05.0 UT (see Eriksson et al., 1994 for time series and spectrograms of this event). At first it may be surprising that no clear increase in the $\boldsymbol{E}$-field for large gradients is seen in panel (A). However, a closer inspection reveals that there is a substantial number of cavities detected during the burst where no enhanced wave activity at all is observed. To investigate the wave-filled cavities further we concentrate on the cases where we see an increase in the electric wave power (blue dots in panel A), which is in approximately half of the cases. In Fig. 5 we see that the electric power for the LHC events can increase almost by a factor of ten. Panel (B) shows that there is no tendency of the magnetic wave power to increase when the density gradients steepen. Even if we consider only observations with a detected increase in electric power (blue dots), we see no tendency toward an enhancement in the magnetic power. This is in accordance with previous experimental studies (Eriksson et al., 1994), as well as with the theoretical model by Hall et al. (2004): even though the inclusion of electromagnetic terms are crucial for understanding LHC wave properties below the lower hybrid frequency, $f_{L H}$, the resulting wave fields are highly electrostatic in the sense of $E / B$ being very large.

\subsubsection{Spatial structures}

To check if the irregular density variations are spatial structures we follow Holmgren and Kintner (1990) and cross correlate two density signals. Event 2 is suitable since we have two density signals that are recorded with a high sampling frequency during several seconds. If $d$ is the probe distance along the satellite trajectory and $\lambda$ is the observed wavelength, the phase difference between the two probes is expressed as

$$
\langle\Delta \phi\rangle=\frac{2 \pi d}{\lambda} .
$$

We see from Eq. (1) that for stationary structures we obtain $\langle\Delta \phi\rangle \approx 0$ in the long wavelength limit. Moreover, for stationary structures, all wavelength components should have equal phase (drift) velocities. If we have stationary structures the frequencies we derive from our time series do not come from a true time variation but are inversely proportional to the wavelength of the static structures. Hence, we obtain $f \lambda=v_{\phi}$, where the phase velocity $v_{\phi}$ is constant and equal to $-v_{s a t}$, where $v_{s a t}$ is the satellite velocity relative to ground.

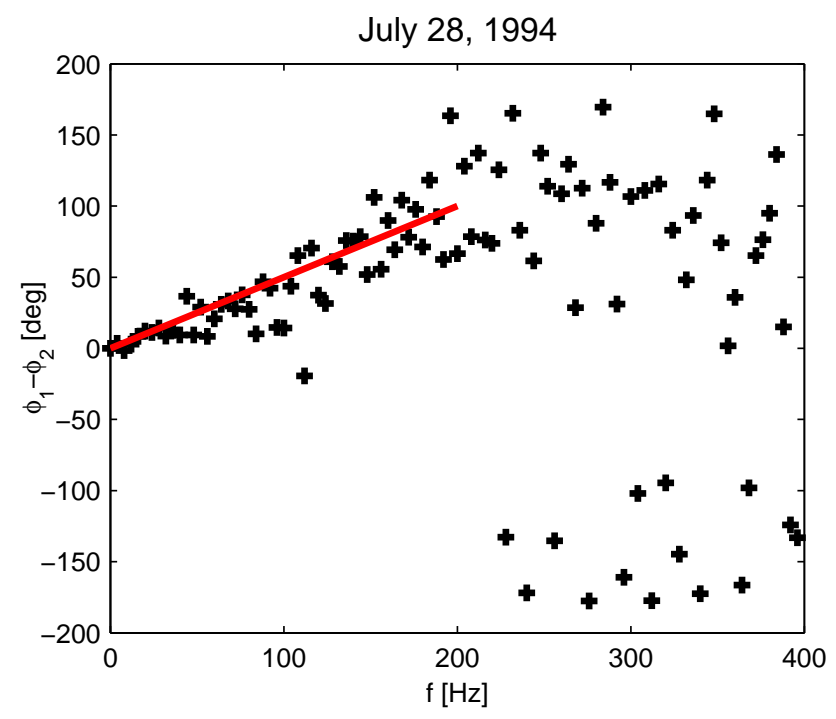

Fig. 6. Phase difference versus frequency for two cross correlated density signals. The phase difference is small in the zero frequency limit. For low frequencies (long wavelengths) there is a good fit to a straight line, and the estimated phase velocity is just the satellite velocity with respect to Earth. The linear fit is made for data with a coherence above 0.5 . Hence, we conclude that the density variations are spatial structures down to scale lengths of a few ion gyroradii $\left(\rho_{H^{+}}=5-10 \mathrm{~m}, \rho_{O^{+}}=20-40 \mathrm{~m}\right)$. Data is taken from the same interval as the data shown in Fig. 3.

To check this assumption we use Eq. (1) to compute the drift velocity as

$v_{\phi}=f \lambda=\frac{2 \pi d f}{\langle\Delta \phi\rangle}$.

Figure 6 presents the phase difference versus frequency. When computing the cross spectrum we used a record length of 1024 points and have averaged over 8 partly overlapping time records. The total time interval used is $0.1 \mathrm{~s}$. For small frequencies (long wavelengths) the linear relationship between the phase difference and the frequency is obvious and both criteria mentioned above are fulfilled.

The linear fit (red line) in Fig. 6 is made only for points with a coherence larger than 0.5 (see Holmgren and Kintner (1990) for details and arguments for doing so). The coherence can be interpreted as the correlation between the signals. From Fig. 6 we obtain a numerical value of $f /\langle\Delta \phi\rangle$. Using the probe separation $d=9 \mathrm{~m}$, Eq. (2) gives $\left|v_{\phi}\right|=7 \mathrm{~km} / \mathrm{s}$, which equals the satellite speed relative to ground.

We notice that the correlation is good for frequencies up to $200 \mathrm{~Hz}$. Since the relative speed between the satellite and the plasma is $7 \mathrm{~km} / \mathrm{s}$, this corresponds to structures larger than $35 \mathrm{~m}$ or an ion gyro radius $\left(\mathrm{O}^{+}, 2 \mathrm{eV}\right)$.

Thus, our analysis strongly indicates that the structures are truly spatial (down to a few ion gyroradii) and that the plasma drift velocity relative to ground is small, at least in the direction parallel to the satellite velocity. 


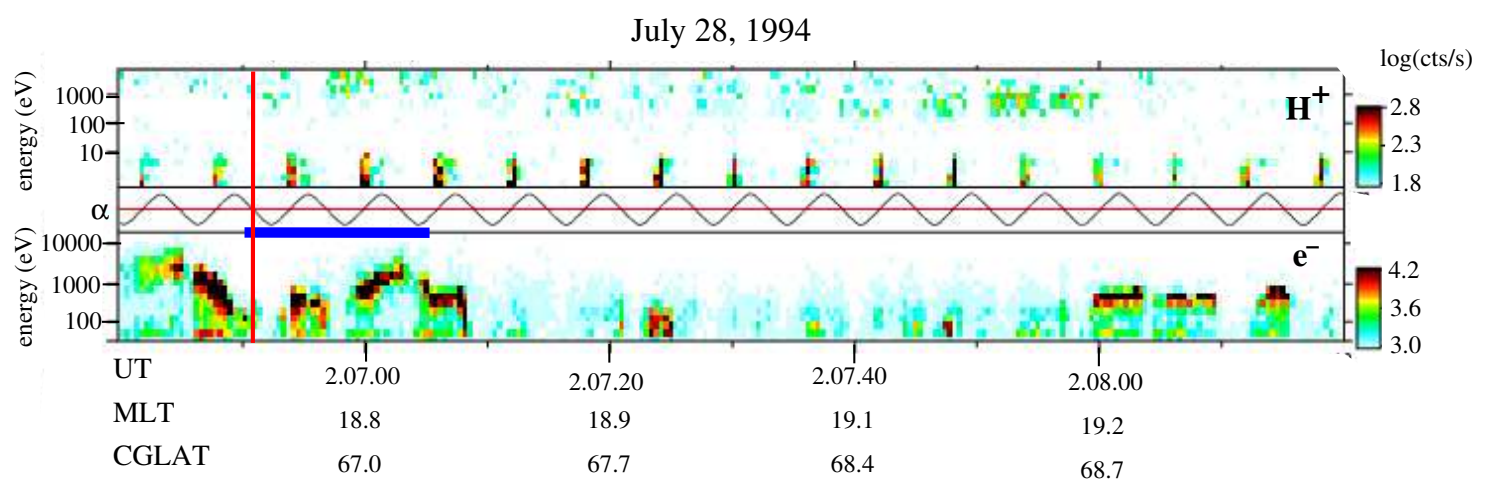

Fig. 7. Particle observations from 28 July 1994. The time interval corresponding to the wave data presented in Fig. 3 is indicated with a thick red line. The top panel shows count rates of $\mathrm{H}^{+}$versus time, while the corresponding pitch angles are displayed in the middle panel. Since the data are recorded in the Northern Hemisphere a pitch angle of $0^{\circ}$ (i.e. parallel to the ambient magnetic field) corresponds to downgoing particles. The bottom panel displays count rates of downgoing electrons. Irregular density variations with enhancements in the wave $\boldsymbol{E}$ - and $\boldsymbol{B}$-fields are found at the edge of an inverted-V structure.

\subsubsection{Particle observations}

Using information from the Freja particle detectors we can characterize the plasma environment in which irregular density variations are found. Figure 7 shows $100 \mathrm{~s}$ of proton and electron overview data. Proton count rates, from the TICS ion spectrometer of the $\mathrm{F} 3 \mathrm{H}$ instrument, obtained by adding data from two neighboring mass spectrometer angular sectors directed nearly perpendicularly to the satellite spin axis, are shown in the top panel. As the spacecraft rotates the detector points in different directions relative to the background magnetic field. The pitch angles of the detected protons are shown in the middle panel. As the observations are made in the Northern Hemisphere, a pitch angle of $0^{\circ}$ corresponds to downgoing protons. The bottom panel presents the downgoing electrons observed by the TESP (F7) detector. Both at the beginning and at the end of the interval, the so-called inverted-V electrons are present. The energy of these downward accelerated electrons is about $1 \mathrm{keV}$. Our data indicate that inverted-V structures are more or less anticorrelated with a weak signature of downgoing protons with $\mathrm{keV}$ energies, suggesting that the spacecraft might be entering a return current region. The low energy ions observed once every satellite spin (every $6 \mathrm{~s}$ ) are heavier ions, mainly $\mathrm{O}^{+}$, recorded due to the satellite ram effect. This is a reoccurring feature in all F3H/TICS data.

The vertical red line in Fig. 7 shows the time of the burst mode wave data previously presented in Fig. 3. The entire burst is 15 s long (02:06:45-02:07:00 UT, marked with a horizontal blue line) and spatial irregular density variations are recorded throughout this time interval. We conclude that density variations with enhanced $\boldsymbol{E}$ - and $\boldsymbol{B}$-fields at the gradients in this case are observed at the edge of an inverted-V electron structure, close to a presumed return current region.

\section{Statistics}

To examine how often irregular density variations and LHCs occur we made a statistical study. As waves above $2 \mathrm{kHz}$ are covered for substantial time intervals only when the F4 instrument is in its burst mode, our data set is limited to all F4 bursts. The total number of available bursts in proper burst modes, with one density measurement, two $\boldsymbol{E}$ - and two $\boldsymbol{B}$-components, is 100 , each $10-15$ s long. The reason for choosing this burst mode is that we need as many field components as possible to study the polarization of the background waves.

To sort out the data of interest we first eliminate all bursts without density variations of at least $1 \%$ over a few ion gyroradii. We also eliminate events with an electric wave amplitude, above the lower hybrid frequency, of less than $1 \mathrm{mV} / \mathrm{m}$. After these eliminations only 13 of the 100 bursts remain. To classify a burst as an LHC event we demand repeated Gaussian-shaped density depletions with widths of $50-100 \mathrm{~m}$. We find that six of our 13 bursts fulfill these criteria. In studies by, for example, Høymork et al. (2000), Dovner et al. (1994), Eriksson et al. (1994), Tjulin et al. (2003), Schuck et al. (2003) and Knudsen et al. (1998) the same criteria have been used to classify LHCs. Six of the remaining seven bursts have density variations that are irregular and do not fulfill the criterias for LHC events. We call these events irregular density variation events. These type of structures are also investigated by, for example, Temerin (1978), Delory et al. (1997), Fejer and Kelley (1980), Sagalyn et al. (1974) and Holmgren and Kintner (1990). Finally, the last burst, Event 1, includes both LHCs and irregular density variations (see Fig. 2). In this statistical study we also include Event 2, which is recorded in a different burst mode giving two density measurements, two $\boldsymbol{E}$ - and one $\boldsymbol{B}$ components. We needed an event with two density measurements to verify that the structures are spatial (see Sect. 3.2.2).

In Table 1 we show some properties of our 14 (13+ Event 2) events. In the first five columns we present the type 
Table 1. Properties of detected irregular density variation events and LHC events. The densities are determined from the plasma frequency in all cases except one. In event 94.06 .05 the density is determined from the $f_{L H}$, where we assumed $95 \% \mathrm{O}^{+}$and $5 \% \mathrm{H}^{+}$. In the seventh column the energetic electrons are characterized. The events where the $\boldsymbol{B}$-field is enhanced on the gradients of the density variations can be seen in the last column. Event 2 from 28 July 1994, is measured in a different burst mode than the rest, with two density signals, two $\boldsymbol{E}$ - and one $\boldsymbol{B}$-component.

\begin{tabular}{|c|c|c|c|c|c|c|c|}
\hline TYPE & $\begin{array}{r}\text { DATE } \\
\text { [yy.mm.dd] }\end{array}$ & $\begin{array}{r}\text { start time } \\
{[\mathrm{UT}]}\end{array}$ & $\begin{array}{r}\text { MLT } \\
\text { [hours] }\end{array}$ & $\begin{array}{r}\text { CGLAT } \\
{\left[{ }^{\circ}\right]}\end{array}$ & $\begin{array}{r}\text { DENSITY } \\
{\left[\# / \mathrm{cm}^{3}\right]}\end{array}$ & Electrons & Enhancement in $\mathbf{B}$ \\
\hline \multirow{7}{*}{$\begin{array}{l}\text { Irregular density variation } \\
\text { events }\end{array}$} & 93.09 .30 & $22: 55: 51.1$ & 17.7 & 66 & 4000 & Inverted-V & Yes \\
\hline & 94.03 .31 & $03: 47: 13.2$ & 23.5 & 67 & 2500 & Inverted-V & Yes \\
\hline & 94.04 .16 & 01:09:12.7 & 21.1 & 72 & 2500 & Inverted-V & Yes \\
\hline & 94.04 .21 & $22: 43: 24.7$ & 18.7 & 67 & 3100 & Inverted-V & Yes \\
\hline & 94.07 .28 & $02: 06: 50.5$ & 18.8 & 67 & 2500 & Inverted-V & Yes \\
\hline & 94.11 .25 & $20: 12: 06.7$ & 21.1 & 59 & 7900 & Radiation belt & No \\
\hline & 95.02 .07 & $05: 18: 12.1$ & 1.6 & 73 & 6000 & Inverted-V & No \\
\hline $\begin{array}{l}\text { Irregular density variation } \\
\text { event/LHC event }\end{array}$ & 94.11 .14 & $22: 30: 57.8$ & 17.9 & 67 & $7900 / 3100$ & Inverted-V & Yes/No \\
\hline \multirow[t]{6}{*}{ LHC events } & 93.09 .03 & 04:45:05.9 & 5.9 & 60 & 1700 & Radiation belt & No \\
\hline & 94.04 .06 & $03: 11: 29.9$ & 21.0 & 64 & 800 & Inverted-V & No \\
\hline & 94.04 .10 & $03: 27: 20.6$ & 19.9 & 64 & 800 & Inverted-V & No \\
\hline & 94.05 .15 & $16: 46: 31.3$ & 11.4 & 57 & 800 & Radiation belt & No \\
\hline & 94.06 .05 & $12: 24: 01.0$ & 13.0 & 61 & 3300 & Radiation belt & No \\
\hline & 95.03 .02 & $23: 26: 59.0$ & 21.0 & 70 & 1300 & Inverted-V & No \\
\hline
\end{tabular}

of density variation, the date of the recorded data, the start time in UT for the bursts, MLT and CGLAT, respectively. The events with enhanced magnetic wave fields at density gradients are listed in the last column. We see that this occurs only for the irregular density variation events. None of our LHC events has enhanced magnetic wave activity at the density gradients, which is expected, since several other studies (see, e.g. Høymork et al., 2000; Dovner et al., 1994; Eriksson et al., 1994) have arrived at the same result. Note that Event 1 (94.11.14) is marked with Yes/No. In the first few seconds of that event we find only irregular density variations with magnetic field enhancements on its gradients. In the latter part there are LHCs, and no density gradient $\boldsymbol{B}$-field enhancements are recorded (see Fig. 2).

In column six we display the electron density, which is estimated from the electron plasma frequency. We find that the density is generally higher for irregular density variation events than for LHC events. In Event 1 the densities are different in regions with irregular density variations and LHCs. This is also reflected in the changing $f_{L H}$ cutoff in panel (A) in Fig. 2.

From the seventh column of Table 1 we see that all irregular density variation events with enhanced magnetic field are related to accelerated auroral electrons. They are generally at the edge of a so-called inverted-V structure, where the electron energies are moderate, rarely above a few $\mathrm{keV}$. Such downgoing electrons are also found during all our LHC events on the nightside, while the LHC events on the dayside at lower latitudes are found in the radiation belt, which is characterized by energetic electrons in the MeV range. Even though we do not have an instrument designed for measuring these electrons we can still easily identify them. They can penetrate the ion instrument and cause an artificial signal evenly spread over all ion energy levels. This is a characteristic signature in the data and easy to identify. For most events we have used the TESP electron data to identify inverted-V electrons (see, e.g. the electron panel in Fig. 7). For a few of the latter events, when TESP was no longer functional, we used data from the MATE electron instrument. MATE have no energy resolution, but we can still, from the time series of the total electron count rate, integrated over the electron energies $0.1-120 \mathrm{keV}$, make a reliable identification of inverted-V events.

To visualize the results from Table 1 we construct Fig. 8, which shows the Northern Hemisphere above $50^{\circ}$ CGLAT, where all the investigated bursts are marked. Irregular density variation events are marked by squares and LHC events by triangles. Filled symbols indicate events with enhanced magnetic wave activity. Event 1 , which includes both irregular density variations and LHCs, is marked by a filled square. Freja bursts, not classified as one of the types above, are marked by dots.

We see from Fig. 8 that irregular density variation events and LHC events are approximately equally common in our 


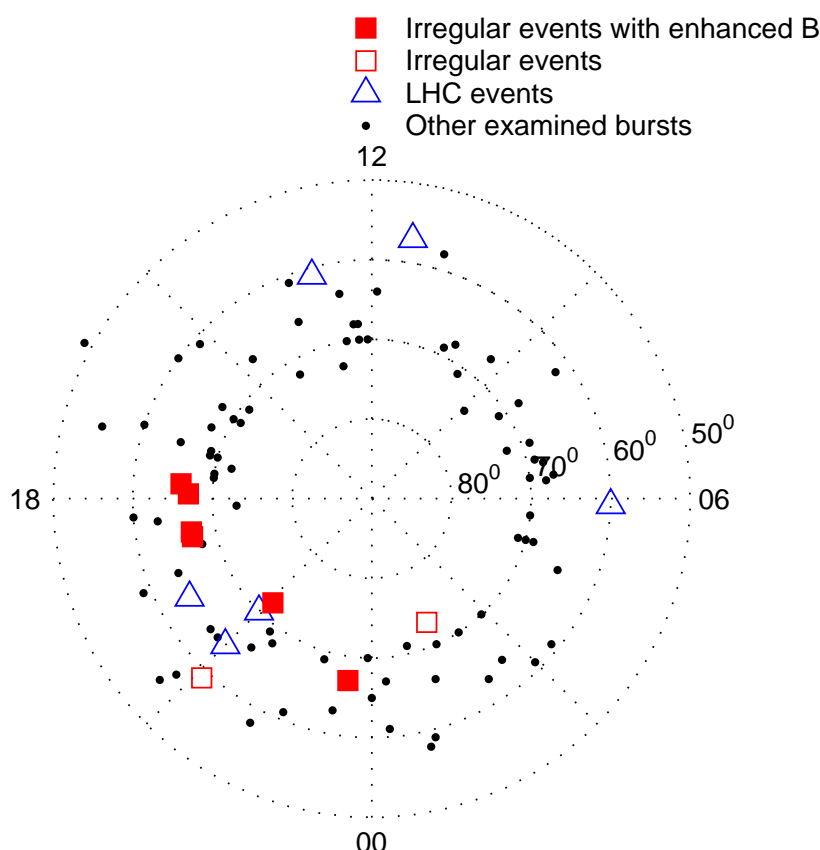

Fig. 8. View over the Northern Hemisphere. The time is given in MLT and the circles indicate the latitude. Due to the $63^{\circ}$ inclination of the Freja orbit we do not measure above $75^{\circ}$. Irregular density variation events are marked by squares. The filled squares indicate where events with enhanced magnetic wave activity have been found. Triangles denote LHC events, while dots mean that no density variations are detected. Event 1 , which includes both irregular density variations and LHCs, is marked by a filled square.

study. The irregular density variation events are located in the pre-midnight sector in the high-latitude auroral region. We find LHC events in the same region but also on the dayside at lower latitudes. This is in agreement with the statistical study of the locations of LHC events at the Freja altitude by Dovner et al. (1997), which was not limited to the F4 bursts and hence based on a much larger data set.

\section{Wave model}

Complete knowledge of the properties of the background waves is important to understand the coupling processes between the gradients/cavities and the waves. Such information is also essential for investigating if interactions between the plasma and the waves are responsible for creating the different kinds of density structures.

We return to Event 1, in order to investigate the nature of the waves in the two different regions there. We model the plasma and use the dispersion solver WHAMP (Rönnmark, 1982). As we aim to describe the waves outside the cavities or gradients, the theory of linear waves in a homogeneous plasma should be applicable. The models used are summarized in Table 2. We assume a quasi-neutral oxygen dominated plasma $\left(\mathrm{O}^{+} 95 \%, \mathrm{H}^{+} 5 \%\right)$, where all ions are nondrifting and cold $(2 \mathrm{eV})$. The electrons are modeled using two
Table 2. The plasma models used in the high $\left(\mathrm{N}_{\mathrm{H}}\right)$ and low density $\left(\mathrm{N}_{\mathrm{L}}\right)$ cases. Four plasma components are used. The background plasma is regarded as cold $(2 \mathrm{eV})$ and oxygen dominated $\left(\mathrm{O}^{+} 95 \%\right.$, $\mathrm{H}^{+} 5 \%$ ). In addition, we assume 5\% downgoing $1 \mathrm{keV}$ inverted$\mathrm{V}$ electrons, whose drift velocity equals the thermal velocity. In Table 2 the drift velocity is normalized to the thermal velocity. The total density is $7900 \mathrm{~cm}^{-3}$ in the high density case and $3100 \mathrm{~cm}^{-3}$ in the low density case. For both cases $f_{H^{+}}=514 \mathrm{~Hz}$.

\begin{tabular}{lrrrrr}
\hline SPECIES & $\begin{array}{r}\mathrm{N}_{\mathrm{H}} \\
{\left[\mathrm{cm}^{-3}\right]}\end{array}$ & $\begin{array}{r}\mathrm{N}_{\mathrm{L}} \\
{\left[\mathrm{cm}^{-3}\right]}\end{array}$ & $\begin{array}{r}\mathrm{T}_{\|} \\
{[\mathrm{eV}]}\end{array}$ & $\begin{array}{r}\mathrm{T}_{\perp} \\
{[\mathrm{eV}]}\end{array}$ & $\begin{array}{r}\text { DRIFT } \\
{[-]}\end{array}$ \\
\hline $\mathrm{H}^{+}$ & 400 & 155 & 2 & 2 & 0 \\
$\mathrm{O}^{+}$ & 7500 & 2945 & 2 & 2 & 0 \\
$\mathrm{e}^{-}$ & 7500 & 2945 & 2 & 2 & 0 \\
$\mathrm{e}^{-}$ & 400 & 155 & 1000 & 1000 & 1 \\
\hline
\end{tabular}

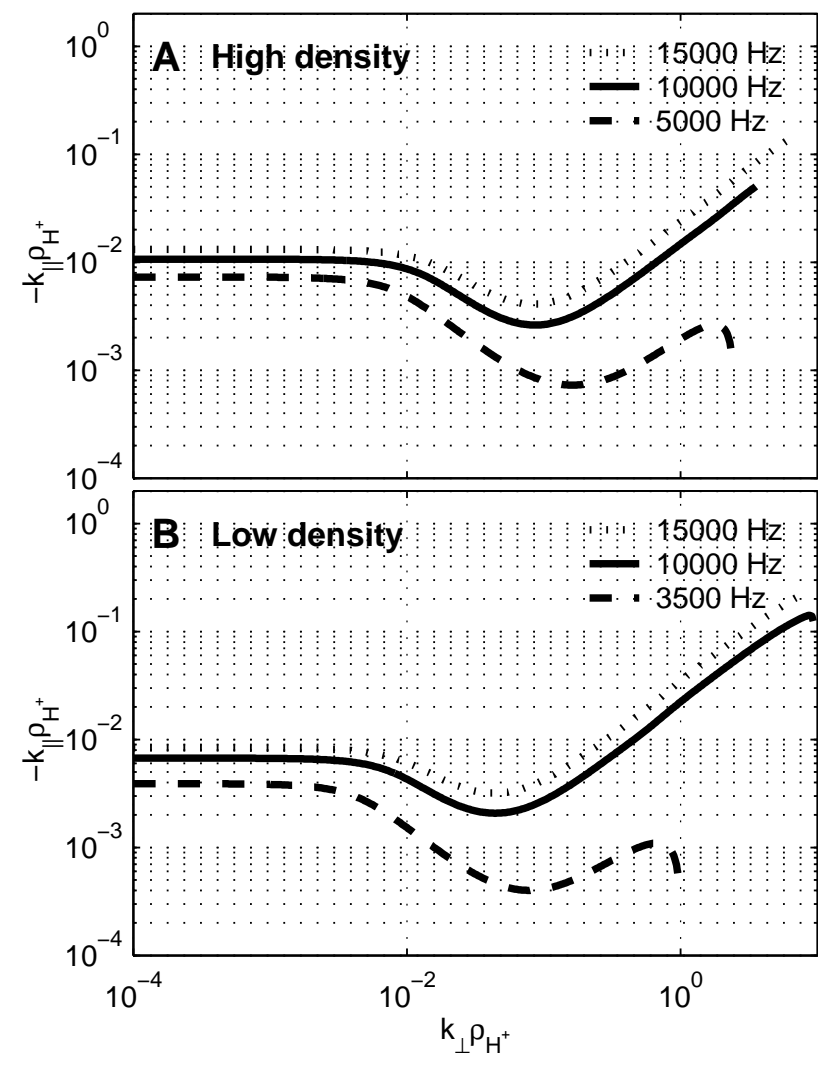

Fig. 9. Constant frequency curves in wave vector space. The parallel and perpendicular wave vectors are normalized to the gyroradius of the $2-\mathrm{eV}$ protons in the models, that is $\rho_{H^{+}}=7 \mathrm{~m}$. Panel (A) shows the results from modelling the high density case, where irregular density events are present. Panel (B) shows the dispersion curves for the low density case. The overall structure is very similar. We can note a small shift in a curve at a given frequency towards larger $k$ for a higher density.

components, a cold ionospheric component and hot inverted$\mathrm{V}$ electrons drifting parallel to $\boldsymbol{B}_{0}$. The drifting electrons constitute $5 \%$ of the total electron density and their drift velocity is equal to their thermal velocity. The total density in 

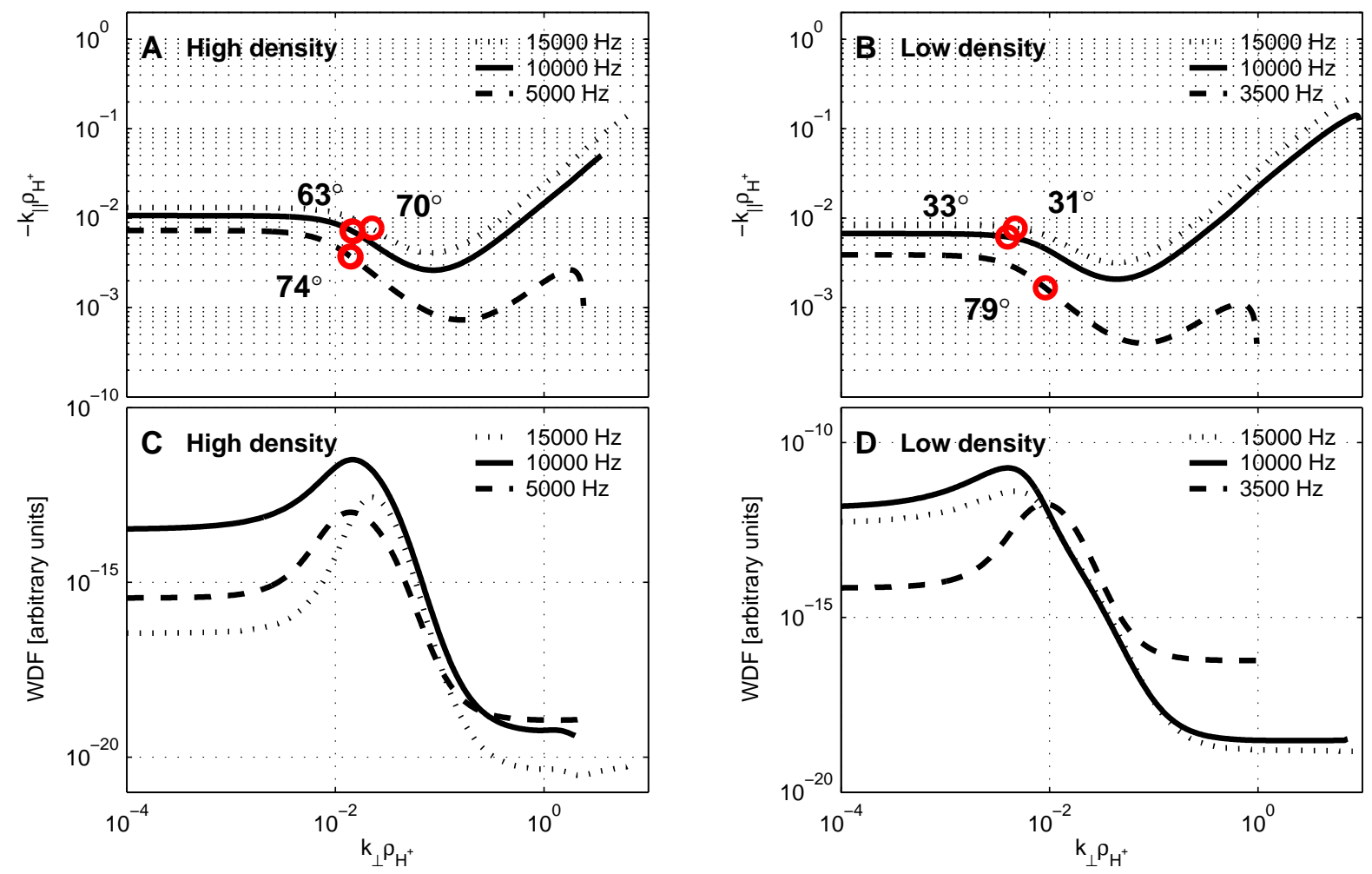

Fig. 10. The peaks in the reconstructed WDF are presented on top of constant frequency curves in the wave vector space in panels (A) and (B). In panels (C) and (D) the WDF is plotted versus normalized $k_{\perp}$. As before $\rho_{H^{+}}=7 \mathrm{~m}$.

the two cases is $3100 \mathrm{~cm}^{-3}$ and $7900 \mathrm{~cm}^{-3}$, respectively. The background magnetic field is approximately $33800 \mathrm{nT}$, giving an electron gyrofrequency of $945 \mathrm{kHz}$. The lower hybrid frequency in the modeled plasma is $3800 \mathrm{~Hz}$ and $6100 \mathrm{~Hz}$ for the two cases, in agreement with Fig. 2. In the frequency range of interest - above the lower hybrid frequency and below both the electron gyrofrequency and the plasma frequency - the only continuous wave mode with a significant magnetic component is the whistler mode. Thus, only this mode is considered in the following analysis.

The mode structure is presented in Fig. 9. At a given frequency an existing wave mode appears as a curve in wave vector $(\boldsymbol{k})$ space. Figure 9 presents three such constant frequency curves in $\boldsymbol{k}$-space for each case. The parallel and perpendicular wave vectors are normalized to the inverse of the gyroradius of the $2 \mathrm{eV}$ protons in the models, that is $\rho_{H^{+}}=7 \mathrm{~m}$. We observe that the overall structure is very similar. Comparing a curve at a given frequency, say $10 \mathrm{kHz}$, in the two different plasmas we note a slight shift in $\boldsymbol{k}$-space.

Polarization parameters, such as $E / B, E_{\perp} / E_{\|}$and $B_{\perp} / B_{\|}$, vary along the constant frequency curves. Hence, by comparing the observations with the theoretical model we can determine where along the curves most of the wave energy is localized. To do this in a systematic way we use Wave Distribution Function (WDF) analysis (Storey and Lefeuvre, 1974; Oscarsson and Rönnmark, 1989). The idea is to use all the available polarization information (e.g. the amplitude and phase relations between the measured field components) to determine the wave energy distribution in wave vector space. Details of the algorithm used can be found in Oscarsson (1994). Stenberg et al. (2002) provide an application of the method. WDF analysis is especially useful in this case, where we only have two electric and two magnetic field components and where conceptually simpler plane wave methods cannot easily be applied.

The results from the WDF analysis are presented in Fig. 10. The constant frequency curves in panels (A) and (B) are the same as in Fig. 9. The red circles on top of these curves denote the peaks in the reconstructed wave energy distributions. For clarity the angle between the wave vector and the background magnetic field is presented next to each peak. We see that the wave energy is most probably located at similar wave vectors in the low and high density cases. In the low density case, waves at higher frequencies $(10$ and $15 \mathrm{kHz})$ propagate more parallel to the ambient field than waves closer to the lower hybrid cutoff frequency $(3.5 \mathrm{kHz})$. This confirms the assumption made in Sect. 3.1 that we observe a mix of a more parallel propagating electromagnetic wave at frequencies around $10 \mathrm{kHz}$ and oblique electrostatic emissions. However, in the high density case the WDF technique does not distinguish between the two wave modes. Waves at all frequencies seem to propagate at a large angle to $\boldsymbol{B}_{0}$. 
Panels (C) and (D) show the reconstructed WDF versus normalized $k_{\perp}$. It is worth noting that the peaks are very pronounced, which indicates that the polarization information in the data is sufficient to pinpoint the wave energy in $\mathbf{k}$-space. From the WDF reconstructions we estimate the perpendicular wavelengths for the high density case to roughly 1500 $4000 \mathrm{~km}$, which is much larger than the small-scale density variations we study. The wavelength is somewhat longer for the low density (LHC) case. From this analysis we find that the waves truly are very similar in the two regions. None of the polarization parameters change much, and the waves are located at similar wave vectors. The perpendicular wavelengths are much longer than the size of the density structures. For both cases examined the waves are found to be electromagnetic whistler mode waves rather than more electrostatic waves located on the resonance cone. Hence, at least in this event, the LHCs are not fed with electrostatic waves, as is often assumed (Schuck et al., 2003).

Finally, we should note that both plasma models used (cf. Table 2) contain a source of free energy, namely the fieldaligned electrons. These electrons are responsible for weak instabilities both at the wave vectors, where we believe the wave energy is located, and at larger perpendicular wave vectors. The location of the instabilities in wave vector space at $10 \mathrm{kHz}$ is shown in Fig. 11. The instabilities at larger $k_{\perp}$ correspond to wavelengths of 70-300 m, which are comparable to the sizes of the density structures. Therefore, since all irregular density variations analyzed in the present study are accompanied by inverted- $\mathrm{V}$ electrons, we cannot exclude a possible connection between the instability and the formation of these density structures. On the other hand, this argument is not valid for LHCs which also exist in the absence of inverted- $\mathrm{V}$ electrons. Therefore, the significance of the instability is unclear.

We conclude that the background waves are similar irrespective of the type of density variations observed. These waves have wavelengths much longer than the typical density structures. It has been suggested that such long wavelength waves can interact with existing small-scale density variations to excite short wavelength electrostatic waves (Bell and Ngo, 1990). Another possibility is that the instability shown in Fig. 11 constitutes the seed for the initiation of the density structures themselves.

\section{Discussion}

In this paper we have studied electric and magnetic waves localized on small-scale density gradients. A number of previous studies of this phenomenon have focused on waves associated with LHCs. We have instead focused on waves found together with irregular density structures. On the gradients of such structures we find enhancements in both the electric and the magnetic wave activity. This is in contrast to most investigations of LHCs, where only the electric power increases in the cavities. A rocket observation reported by D. Knudsen (private communication) is, as far as we know, the only case

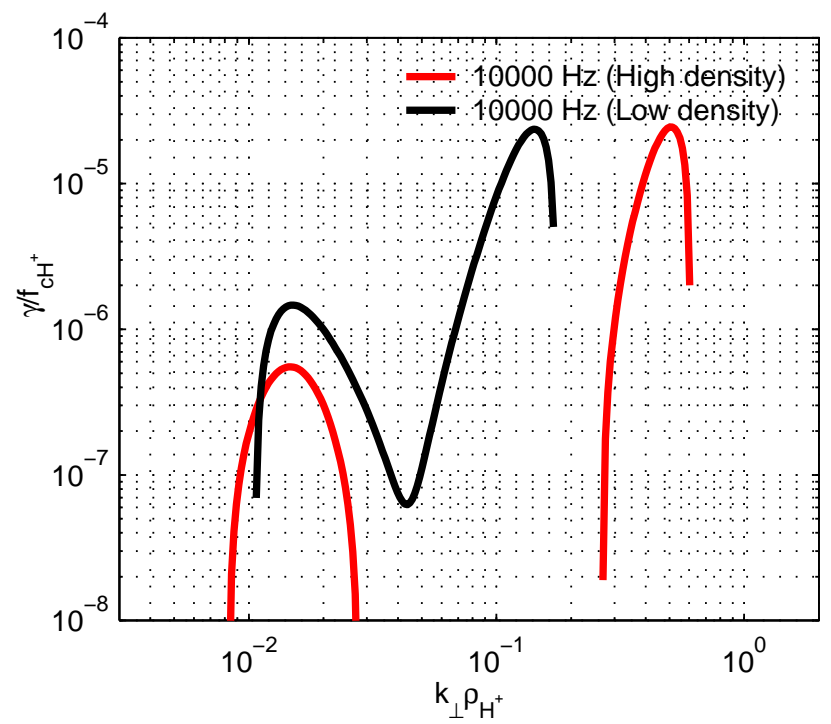

Fig. 11. The temporal growth rate versus $k_{\perp} \rho_{H}^{+}\left(\rho_{H^{+}}=7 \mathrm{~m}\right)$. In both the high (red lines) and low density (black line) cases there are two peaks in the growth rate, one at the perpendicular wave vector, where we believe most of the wave energy to be located, and one at larger $k_{\perp}$.

where there are also enhancements in the magnetic wave activity associated with LHCs.

We have found that the densities are considerably higher for irregular density variation events than for LHC events. Also, the shape of the density variations differs between the two event types. These two features might explain the difference in nature between the enhanced waves on the density gradients.

Furthermore, we see from Figs. 4 and 5 that the electric field almost always is enhanced on the large gradients of the irregular density variations, but only on about half of the large LHC gradients. However, we have examined only a few events and the confirmation of the observations will be included in a future study.

The presence of inverted-V electrons near the irregular density variations suggests that whistler waves are at least marginally unstable near $10 \mathrm{kHz}$. Hence, the narrow band emission shown in panel (b) in Fig. 2 may be caused by a space plasma instability, but since man-made signals are ubiquitous in the magnetosphere we cannot rule out that it originated from a ground transmitter. However, the origin of the waves should not affect how they interact with the irregular density variations. In particular, the enhancement of the wave magnetic field on the gradients of the irregular density variations are not explained by assuming that the waves come from a ground-based radio transmitter.

In laboratory experiments, waves localized in density depletions have $\nabla \times \boldsymbol{E} \neq 0$. Thus, they are electromagnetic (see, for example, Rosenberg and Gekelman, 2001). There are discussions about whether the conditions in the laboratories are similar to those for LHCs in space (Schuck et al., 2004; 
Gekelman, 2004). The shape of the depletions are somewhat different. This might explain the different nature of LHCs and waves measured in laboratories. The conditions in laboratories are in certain respects more similar to irregular density variation events. We do not claim that the waves in laboratories are of the same type as the waves we measure but do not exclude that they are related.

\section{Conclusions}

In this paper we focus on small-scale irregular density variations observed by the Freja satellite at $1000-1700 \mathrm{~km}$ in the auroral zone. The main conclusions are summarized below:

- Irregular density variations in an environment of whistler mode/lower hybrid waves are quite common at the Freja altitude. The variations we observe are spatial structures with a very low drift velocity. If the gradients of the density structures are steep enough, both the electric and magnetic wave fields are usually enhanced.

- In a small statistical study we find that irregular density variations and LHCs occur equally as often. Generally, the observed densities are higher when we record irregular density variations than when LHCs are detected.

- Gradients with enhanced electric and magnetic wave fields are found in the pre-midnight sector (MLT 18:0000:00). Generally, they are found at the edges of inverted- $\mathrm{V}$ electron structures, and sometimes very close to return current regions, indicated by downgoing $\mathrm{keV}$ protons.

- The background waves are electromagnetic $\left(E / B \approx 5 \cdot 10^{7} \mathrm{~m} / \mathrm{s}\right.$ at $\left.10 \mathrm{kHz}\right)$ whistler mode waves. The wavelengths are of the order of 1.5-4.0 km, which is much larger than the typical size of the density structures. At the gradients we observe a significant increase in $E / B$, indicating that the localized waves have much shorter wavelengths. Similar electromagnetic background waves are also observed in the LHC case investigated.

Acknowledgements. The authors thanks J. O. Hall and H. Pécseli for useful discussions. We also acknowledge Freja PIs for providing data. A. Reiniusson is supported by the Swedish National Graduate School of Space Technology.

Topical Editor I. A. Daglis thanks two referees for their help in evaluating this paper.

\section{References}

André, M. (Ed.): The Freja scientific satellite, IRF Scientific Report 214, Swedish Institute of Space Physics, Kiruna, 1993.

Bamber, J. F., Maggs, J. E., and Gekelman, W.: Whistler wave interaction with a density striation: A laboratory investigation of an auroral process, J. Geophys. Res., 100, 23 795-23 810, 1995.
Bell, T. F. and Ngo, H. D.: Electrostatic lower hybrid waves exicited by electromagnetic whistler mode waves scattering from planar magnetic-field-aligned plasma density irregularities., J. Geophys. Res., 95, 149-172, 1990.

Boehm, M., Paschmann, G., Clemmons, J., et al.: The TESP electron spectrometer and correlator (F7) on Freja, Space Sci. Rev., 70, 509-540, 1994

Delory, G. T., Ergun, R. E., Klementis, E. M., et al.: Measurements of short wavelength VLF bursts in the auroral ionosphere: A case for electromagnetic mode conversion?, Geophys. Res. Lett., 24, 1131-1134, 1997.

Dovner, P. O., Eriksson, A. I., Boström, R., et al.: Geophys. Res. Lett., 21, 1827-1830, 1994.

Dovner, P. O., Eriksson, A. I., Boström, R., et al.: Geophys. Res. Lett. 24, 619-622, 1997.

Eliasson, L., Norberg, O., Lundin, R., et al.: The Freja hot plasma experiment: Instrument and first results, Space Sci. Rev., 70, 563-576, 1994.

Eriksson, A. I., Holback B., Dovner, P. O., et al.: Geophys. Res. Lett., 21, 1843-1846, 1994.

Eriksson, A. and Boström, R.: Wave measurements using electrostatic probes: accuracy evaluation by means of a multiprobe technique, Measurement techniques in space plasmas: Fields (AGU geophysical monograph 103), American Geophysical Union, 147-153, 1998.

Gekelman, W.: Comment on "Properties of lower hybrid solitary structures: A comparison between space observations, a laboratory experiment and the cold homogeneous plasma dispersion relation" by Schuck et al., J. Geophys. Res., 109, doi:10.1029/2002JA009778, 2004.

Green, James L., Boardsen S., Garcia L., et al.: On the origin of whistler mode radiation in the plasmasphere, J. Geophys. Res., 110, doi:10.1029/2004JA010495, 2005.

Fejer, B. G. and Kelley, M. C.: Ionospheric irregularities, Rev. Geophys., 18, 401-454, 1980.

Hall, J. O., Eriksson, A. I., and Leyser, T. B.: Excitation of localized rotating waves in plasma density cavities by scattering of fast magnetosonic waves, Phys. Rev. Lett. 92, Art. No. 255002, 2004.

Holmgren, G. and Kintner, P. M.: Experimental Evidence of Widespread Regions of Small-Scale Plasma Irregularities in the Magnetosphere, J. Geophys. Res., 95, 6015-6023, 1990.

Holback, B., Jansson, S.-E., Åhlén, L., et al.: The Freja wave and plasma density experiment, Space Sci. Rev., 70, 577-592, 1994.

Høymork, S. H., Pécseli, H. L., Lybekk, B., et al.: Cavitation of Lower-Hybrid Waves in the Earth's ionosphere; a model analysis, J. Geophys. Res., 105, 18 519-18 535, 2000.

Kimura, I., Kasahara, Y., and Oya, H.: Determination of global plasmaspheric electron density profile by tomographic approach using omega signals and ray tracing, J. Atmos. S.-P., 63, 11571170, 2001.

Knudsen, D. J., Dovner, P. O., Eriksson, A. I., et al.: Effect of lower hybrid cavities on core plasma observed by Freja, J. Geophys. Res., 103, 4241-4249, 1998.

Lundin, R., Haerendel, G., and Grahn, S.: The Freja Project, Geophys. Res. Lett., 21, 1823-1826, 1994a.

Lundin, R., Haerendel, G., and Grahn, S.: The Freja science mission, Space Sci. rev., 70, 405-419, 1994b.

Lundin, R., Haerendel, G., and Grahn, S.: Introduction to special section: The Freja mission, J. Geophys. Res., 103, 4119-4123, 1998.

Oscarsson, T.: Dual Principles in Maximum Entropy Reconstruction of the Wave Distribution Function, J. Comput. Phys., 110, 
221-233, 1994.

Oscarsson, T. and Rönnmark, K.: Reconstruction of wave distribution functions in warm plasmas, J. Geophys. Res., 94, 2417 2427, 1989.

Rosenberg, S. and Gekelman, W.: A three-dimensional experimental study of lower hybrid wave interactions with field-aligned density depletions, J. Geophys. Res., 106, 28 867-28 884, 2001.

Rönnmark, K.: WHAMP - Waves in Homogeneous Anisotropic Multicomponent Plasmas, KGI Report 179, Kiruna Geophysical Institute, 1982.

Sagalyn, R. C., Smitty, M., and Ahmed, M.: High-latitude irregularities in the topside ionosphere based on Isis 1 thermal probe data, J. Geophys. Res., 79, 4252-4261, 1974.

Schuck, P. W., Bonnell, J. W., and Kintner, P. M.: A Review of Lower Hybrid Solitary Structures, IEEE Trans. Plasma Sci., 31, 1125-1177, 2003.

Schuck, P. W., Bonnell, J. W., and Pinçon, J.-L.: Properties of lower hybrid solitary structures: A comparison between space observations, a laboratory experiment and the cold homogeneous plasma dispersion relation, J. Geophys. Res., 109, doi:10.1029/2002JA009673, 2004.
Sonwalkar, V. S., and Harikumar, J.: An explanation of ground observations of auroral hiss: Role of density depletions and meterscale irregularities, J. Geophys. Res., 105, 18 867-18 883, 2000.

Stenberg, G., Oscarsson, T., André, M., et al.: Investigating wave data from the FAST satellite by reconstructing the wave distribution function, J. Geophys. Res., 107, doi: 10.1029/2001/2001JA900154, 2002.

Storey, L. R. O., and Lefeuvre, F.: Theory for the interpretation of measurements of the six components of a random electromagnetic wave field in space, Space Res., 14, 381-386, 1974.

Temerin, M.: The polarization, frequency and wavelengths of highlatitude turbulence, J. Geophys. Res., 83, 2609-2616, 1978.

Tjulin, A., Eriksson, A. I., and André, M.: Lower hybrid cavities in the inner magnetosphere, Geophys. Res, Lett., 30, doi:10.1029/2003GL016915, 2003.

Zanetti, L. and the Freja Magnetic Field Experiment Team: Magnetic field experiment on the Freja satellite, Space Sci. Rev., 70 , 465-482, 1994. 